\title{
Development of a drop-on-demand droplet generator for one-drop-fill technology
}

\author{
Kuang-Chao Fan ${ }^{\mathrm{a}, *}$, Jhih-Yuan Chen ${ }^{\mathrm{a}}$, Ching-Hua Wang ${ }^{\mathrm{a}}$, Wen-Chueh Pan ${ }^{\mathrm{b}}$ \\ a Department of Mechanical Engineering, National Taiwan University, Taipei, Taiwan, ROC \\ ${ }^{\mathrm{b}}$ Chung-Shan Institute of Science \& Technology, Taiwan, ROC
}

\section{A R T I C L E I N F O}

\section{Article history:}

Received 4 December 2007

Received in revised form 20 February 2008

Accepted 3 March 2008

Available online 14 March 2008

\section{Keywords:}

Droplet generator

One-drop-fill

Drop-on-demand

Liquid crystal

\section{Introduction}

The manufacturing of liquid crystal displays (LCD) is divided into three processes: the array process, the cell process and the module process. When the panel size is beyond the fifth generation $(1120 \mathrm{~mm} \times 1250 \mathrm{~mm})$, the liquid crystal (LC) filling process undergoes a technological change, namely the one-drop-fill (ODF) technology, i.e. from the conventional injection method between two glass substrates to the novel dispensing method onto the bottom substrate and pressing down the upper substrate [1-3]. The main advantage is to largely reduce the liquid crystal filling up time. For example, with the conventional injection method it takes 2 days to fill up a 22-in. panel, but with the ODF method this task can be completed within $2 \mathrm{~h}$ only.

The key of the ODF technology is to use a dispenser for controlling the amount of liquid crystal material deposited on the substrate. Many methods have already been proposed [4-6]. A typical one is to control the valve and driving source, e.g. the gas or the plunger, in such a way that the demanded liquid can be dropped on the prescribed location of the substrate. The liquid crystal material has to be kept pure during the dispensing process. As the motion of the actuator increases, the possibility to pollute the LC increases. For example, when the valve or the plunger is actuated, metal particles will be produced by rubbing and cause the liquid crystal material to be polluted.

\footnotetext{
* Corresponding author. Tel.: +88622362 0032; fax: +886223641186 E-mail addresses: fan@ntu.edu.tw (K.-C. Fan), d93522025@ntu.edu.tw (J.-Y. Chen).
}

The inkjet printing technology has been widely used in many fields, such as MEMS, electronics manufacturing [7], PLED [8], RP [9] and biosensor applications [10], due to its advantages of fast speed, high resolution and high accuracy. In this research, the basics of the inkjet principle are used to develop the ODF technology.

\section{Development of a droplet generator}

The inkjet printing technology can commonly be divided into two classes: the continuous mode [11] and the drop-on-demand (DOD) mode [12]. In recent years, the DOD mode has gradually replaced the continuous mode because of promising better manufacturing control. The droplet is ejected by applying a voltage pulse to the piezoelectric actuator so that its ejection time, position and volume can be easily controlled by a function generator.

There are several types of droplet generators depending on four piezoelectric material deformation modes, namely, the squeeze mode [13,14], the shear mode [15], the bend mode [16] and the push mode. For designing the simple structure of a low cost droplet generator, we use a disk-type piezoelectric buzzer as driving source and the PZT motion is equivalent to the bend mode of the droplet generator. Several development steps are described in the followings.

\subsection{Structure of the droplet generator}

Liquid crystal material is a pure chemical compound. For all LCD manufacturers, it is a serious subject to prevent the liquid crystal material from being contaminated. There are many factors to be considered, such as environment, human errors and metal particles 


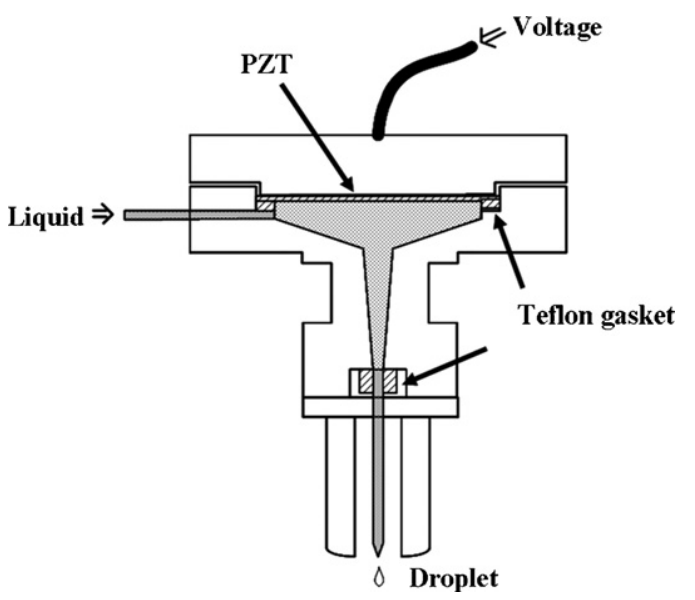

Fig. 1. Assembly of the droplet generator.

produced by rubbing. In addition, the proper selection of contact materials must also be taken into account, in case some chemical reactions take place.

Fig. 1 shows the assembly of the droplet generator. The piezoelectric buzzer is constructed of a thin piezo-ceramic layer, about $0.2-\mathrm{mm}$ thick, which sticks on a vibration diaphragm with a $27-$ $\mathrm{mm}$ diameter. It is fixed within the main body and bends if a voltage pulse is applied. The pressure is generated in the liquid flow channel and pushes the liquid out of the glass nozzle. The structure of the proposed droplet generator consists of five main parts: (1) main body with liquid flow channel and upper cover, (2) piezoelectric buzzer, (3) Teflon gasket, (4) nozzle holder and (5) glass nozzle, as shown in Fig. 2. The selected materials are stainless steel, Teflon and glass because these three materials are apt to contact with liquid crystal.

\subsection{Fabrication of the glass nozzles}

Forming stable droplets is an important issue for all droplet generators. During the dispensing process, the quality of the glass nozzle directly affects the droplet formation process. Skewing or unstable formation of droplets may be caused, if liquids with high viscosity or high surface tension are subject to adhere to the front surface of the nozzle.

We chose glass to make the nozzle because of several advantages, such as good chemical resistance, low friction, easy to

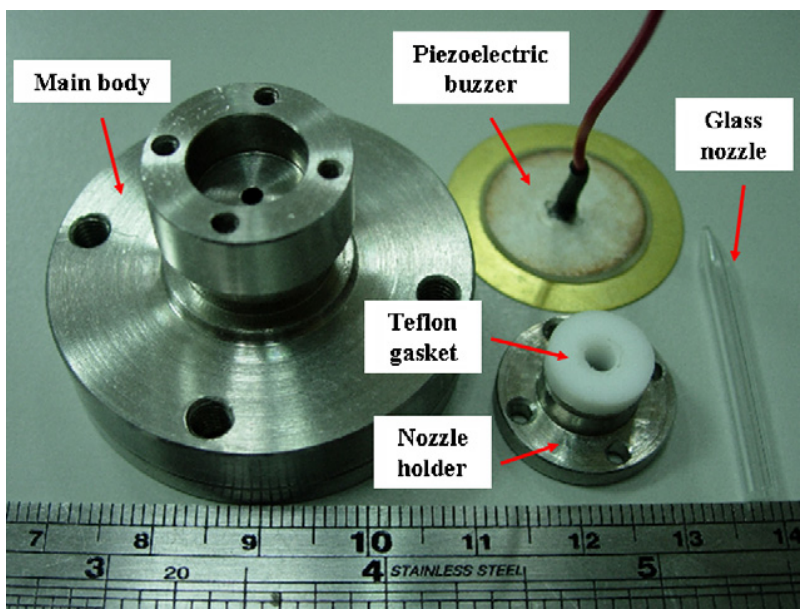

Fig. 2. Photo of the developed droplet generator.

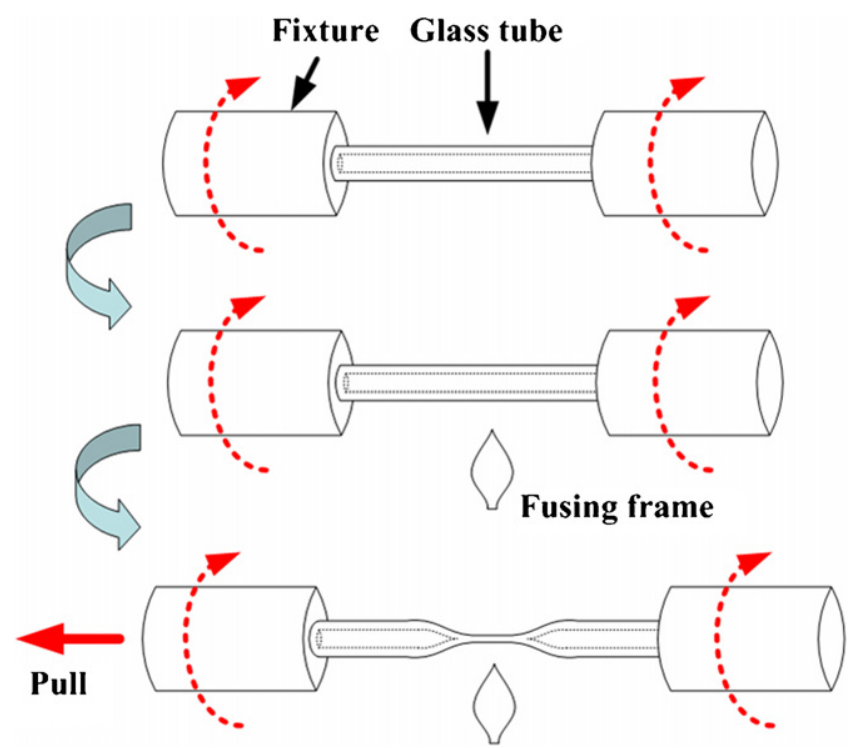

Fig. 3. Fabrication process of the glass nozzle.

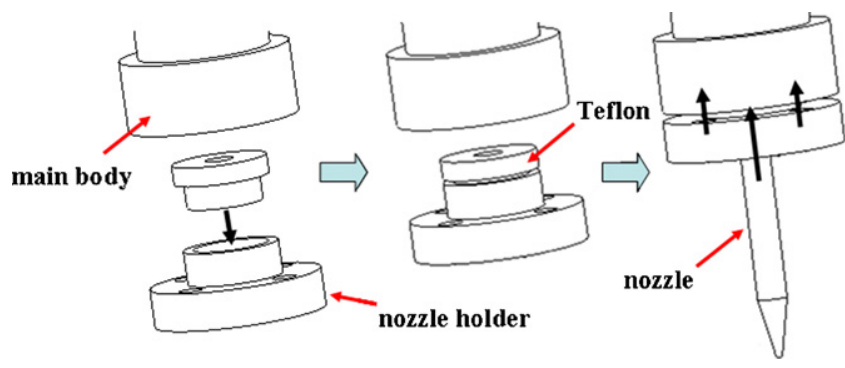

Fig. 4. Assembling process.

manufacture and low cost. In the fabrication process, the glass tube is fixed at both ends and then rotated within the coaxial fixtures, as shown in Fig. 3. The central part of the glass tube is heated by a fusing flame until the temperature exceeds transition temperature of the glass. By pulling outward one side of the fixtures, the softened part of glass tube will be necked off, forming a sharp taper. Finally, cutting it off, we get two unfinished glass nozzles. The end surface of the nozzle must be ground after the glass fusing process, in order to obtain the required diameter and a round opening of the nozzle head. The shape of the nozzle tip and the smoothness of the front face must be assured. Otherwise, poor droplet formation and skewed path will occur [16,17].

\subsection{Assembling and dismantling}

At the end of each liquid filling process, the remaining liquid has to be drained out and the parts have to be cleaned. Fig. 4 shows that a Teflon gasket is particularly designed to connect the main body and the nozzle holder. When pushing the glass nozzle into the nozzle holder, the Teflon gasket will clip the glass nozzle after locking the nozzle holder. This design helps the dismantling process to be done easily and ensures the assembled structure to be fully sealed.

\section{Experimental set-up}

The goal of this research is to provide a method to dispense an optimum quantity of liquid crystal material on the glass substrate during the LCD cell process. Therefore it is necessary to measure the volume of the droplet. The on-line measurement system is composed of the drop-on-demand system and the stroboscopic 


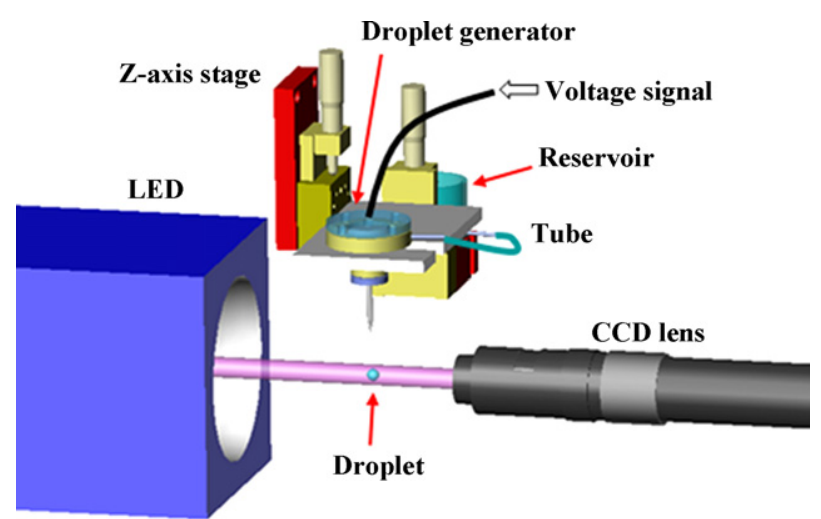

Fig. 5. On-line measurement system.

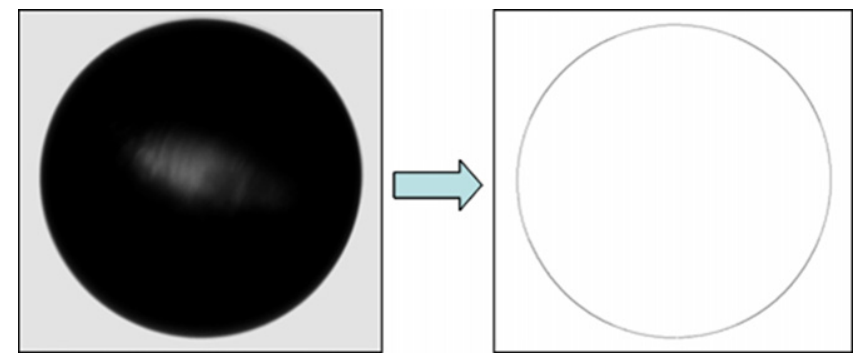

Fig. 6. To estimate the diameter of the droplet by image processing method.

technique. As shown in Fig. 5, a tube is used to connect the droplet generator and the reservoir which supplies the LC material and keeps the back pressure steady. In order to generate constant pressure waves, the whole volume of the flow channel must be filled up with LC material. A voltage pattern is generated by a function generator to drive the droplet generator via a power amplifier. The output voltage to the PZT is adjustable between $20 \mathrm{~V}$ and $100 \mathrm{~V}$. In this DOD system, the waveform of the function generator can be tuned, in order to control the number and the size of the droplet [18]. An XY stage can move the droplet generator to the prescribed positions, in order to arrange the LC drop in an array pattern on the substrate. The stroboscopic technique has been applied by mounting a lightemitting diode (LED) and a CCD camera. The LED is simultaneously triggered by the same frequency as the function generator actuates the PZT film. A frozen image of the droplet can thus be viewed and captured by the CCD camera. Finally, we can utilize the image processing method and the least square fitting of planar circles to estimate the diameter of the droplet, as shown in Fig. 6. The control software is developed by using Microsoft Visual C++ 6.0.

\section{Experimental results}

In our research, we investigated the phenomenon of dispensing, in order to improve the accuracy and reliability of the droplet generator. There are three process parameters to be kept under control, namely, the quality of the nozzle, the voltage signals and the liquid properties. We expected to find out the best control parameters of the actuator in order to precisely control the total amount of the liquid crystal material.

\subsection{Glass nozzle}

The roundness of the nozzle head will affect the degree of the slant distance of the droplet. Some factors such as machine vibrations and high grinding forces will cause the opening of the glass nozzle with a diameter of approximately $320 \mu \mathrm{m}$ to be breached, (a)

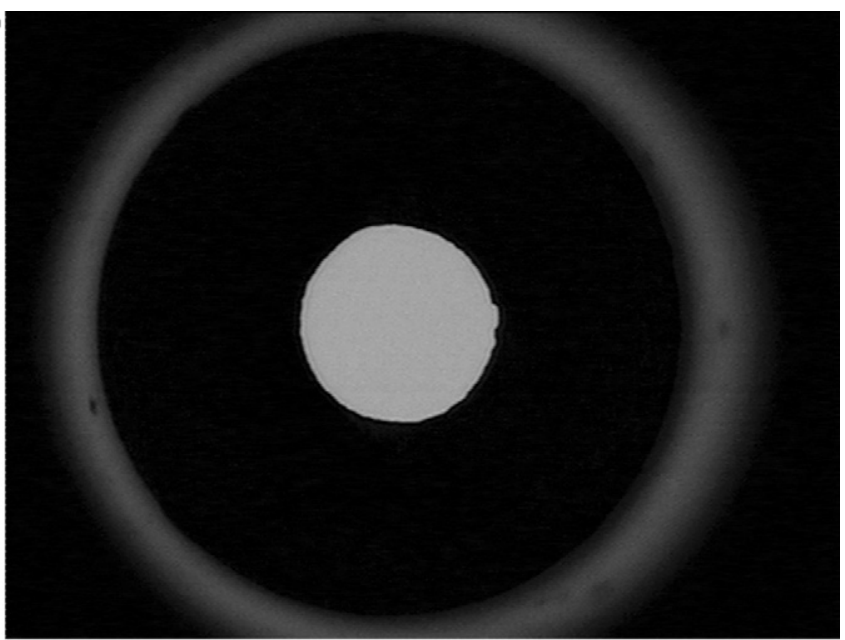

(b)

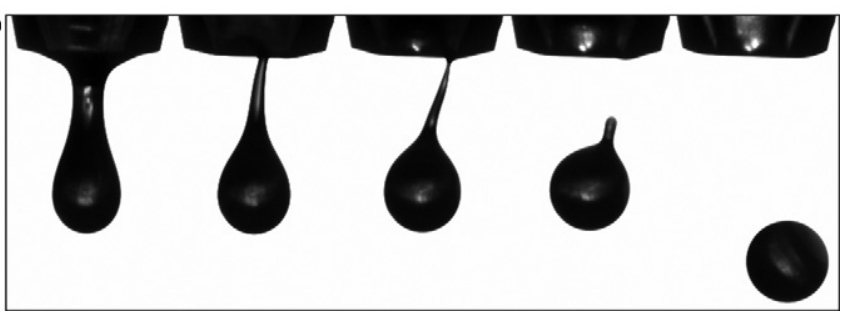

Fig. 7. (a) Breached nozzle head and (b) a sequence of the drop formation.

as shown in Fig. 7a. Such a breach will result in a skewed liquid pattern after ejecting and shown in Fig. 7b. Thus, more attention must be paid to the grinding process. Fig. $8 \mathrm{a}$ and $\mathrm{b}$ shows a good nozzle head with a diameter of approximately $300 \mu \mathrm{m}$ and a sequence of the stable drop formation.

Fig. 9a visualized the definition of flying distance and slant distance. Six nozzle heads were tested and listed in Table 1 . The tested

(a)

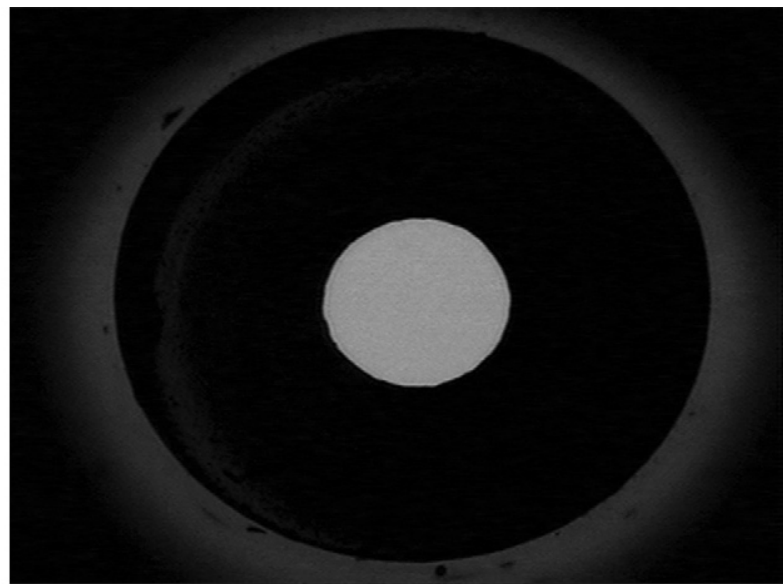

(b)

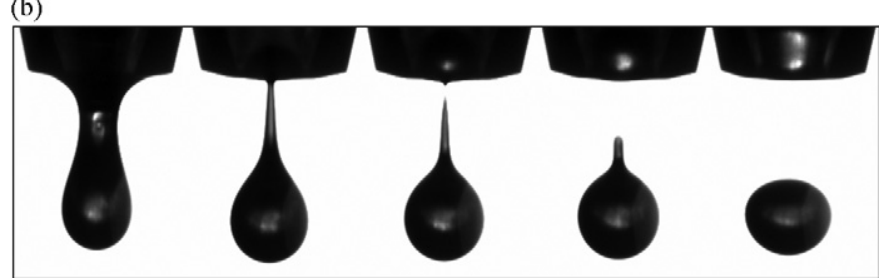

Fig. 8. (a) Good nozzle head and (b) a sequence of the drop formation. 

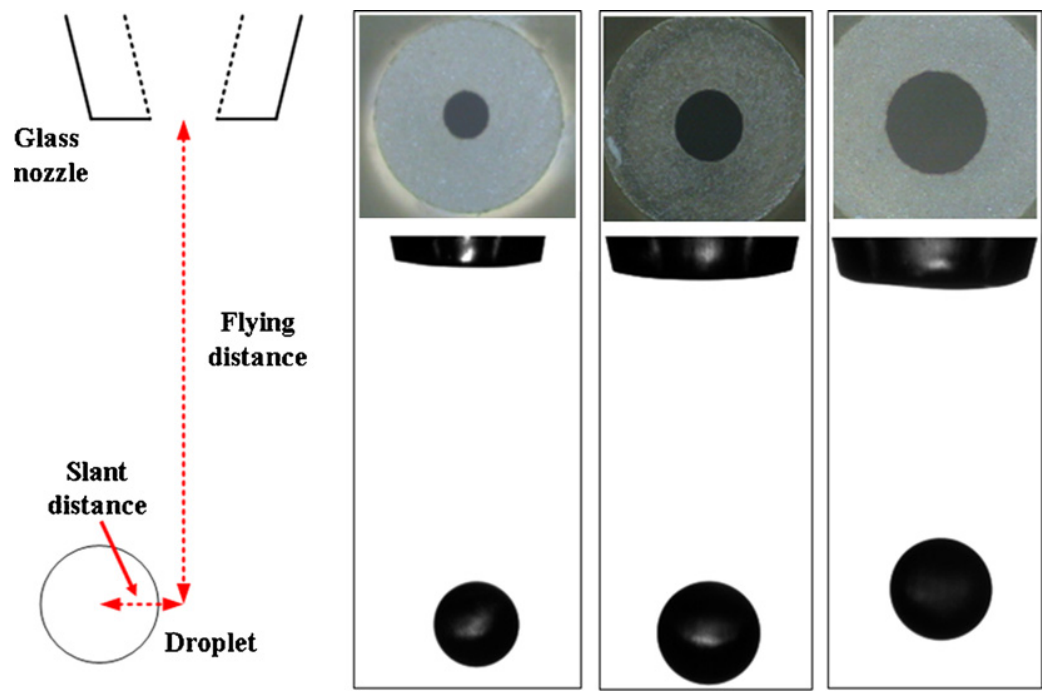

Fig. 9. (a) Definition of flying distance and slant distance. Ejection behavior of three different nozzle sizes: (b) $197.6 \mu \mathrm{m}$, (c) $300.2 \mu \mathrm{m}$ and (d) $410.5 \mu \mathrm{m}$.

Table 1

Measurement of the slant distance by varying the nozzle sizes and its roundness

\begin{tabular}{|c|c|c|c|c|c|}
\hline No. & Nozzle size $(\mu \mathrm{m})$ & Roundness $(\mu \mathrm{m})$ & Roundness/nozzle size & Slant distance $(\mu \mathrm{m})$ & Flying distance $(\mu \mathrm{m})$ \\
\hline 1 & 197.6 & 5.3 & 0.027 & 50.1 & 2053 \\
\hline 2 & 300.2 & 6.0 & 0.02 & 36.6 & 1977 \\
\hline 3 & 410.5 & 9.5 & 0.023 & 43.4 & 1743 \\
\hline 4 & 152.13 & 5.34 & 0.035 & 103.8 & 2123 \\
\hline 5 & 273.38 & 9.53 & 0.035 & 99.9 & 2078 \\
\hline 6 & 347.97 & 17.88 & 0.051 & 196.8 & 2150 \\
\hline
\end{tabular}

liquid is liquid crystal (Chisso, ZOC-5114LA) and its viscosity is $17.4 \mathrm{mPa}$ s. If the ratio between the roundness and nozzle size was larger than 0.035 , an unwanted slant distance which was larger than $100 \mu \mathrm{m}$ when the flying distance approximate $2 \mathrm{~mm}$ was usually obtained. This could be an indicator to improve the droplet skewing. The smaller slant distance could be obtained.

Fig. 10 shows the relationship between the nozzle size and the drop size. As the nozzle size increases, the droplet size increases linearly at the same driving voltage. The corresponding range of drop volumes is between $11 \mathrm{~nL}$ and $66 \mathrm{~nL}$.

\subsection{Voltage signal}

Fig. 11 shows the input parameters of the voltage, including the rising time (t_rise), the dwell time ( $\left.t \_d w e l l\right)$, falling time ( $\left.t \_f a l l\right)$,

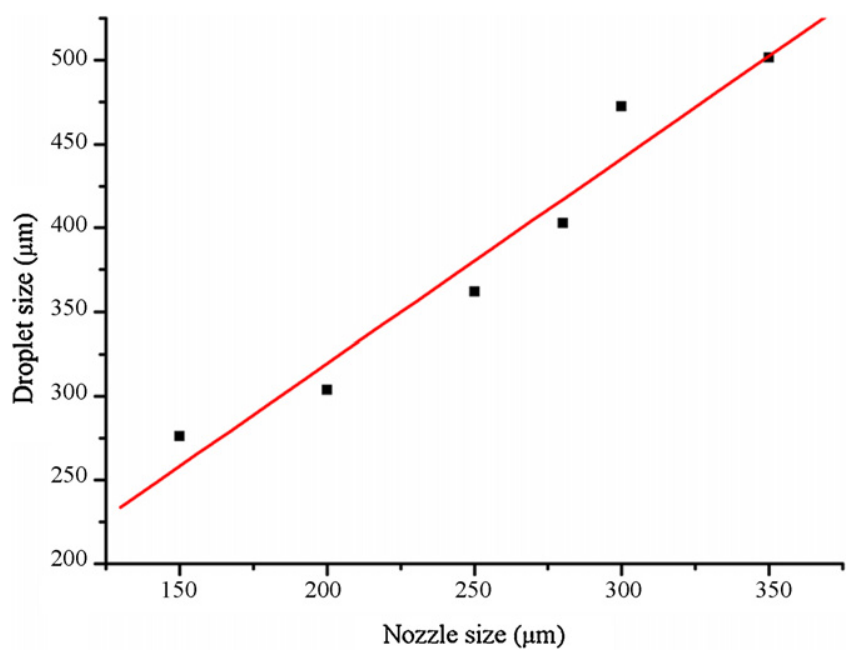

Fig. 10. Effect of nozzle size on drop size. pulse width (t_width) and amplitude of the voltage (V). Here, t_rise is always set to be greater than $1 \mu \mathrm{s}$. In practice, we use t_rise $=10 \mu \mathrm{s}$ and $t_{\_}$fall $=10 \mu \mathrm{s}$.

\subsubsection{Jetting frequency}

According to the propagation and reflection of pressure waves produced by the PZT vibration [19], the liquid is pushed through the nozzle at an optimal time. After the droplet has been formed, the pressure waves are reflected in the cavity. In order to obtain a steady drop formation, the next pulse of PZT has to wait until the last waves are fully decayed. In other words, the highest working frequency of PZT will be limited by the decay-speed of the pressure waves. From the experiments, a stable condition below $50 \mathrm{~Hz}$ has been confirmed by the stability of the drop volume with a variation of about $3.2 \mathrm{~nL}$ only, as shown in Fig. 12. The used nozzle size was $350 \mu \mathrm{m}$.

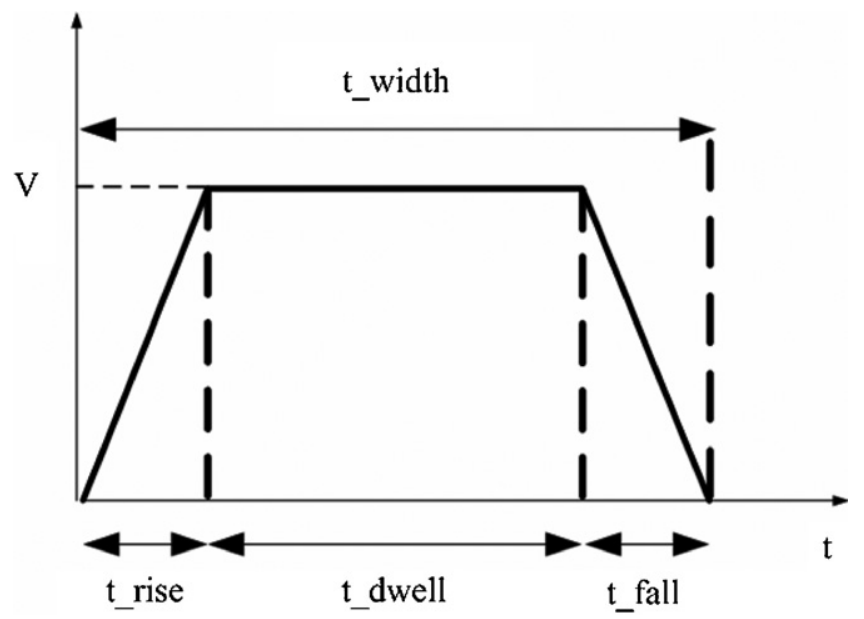

Fig. 11. The wave form of the voltage at the PZT. 


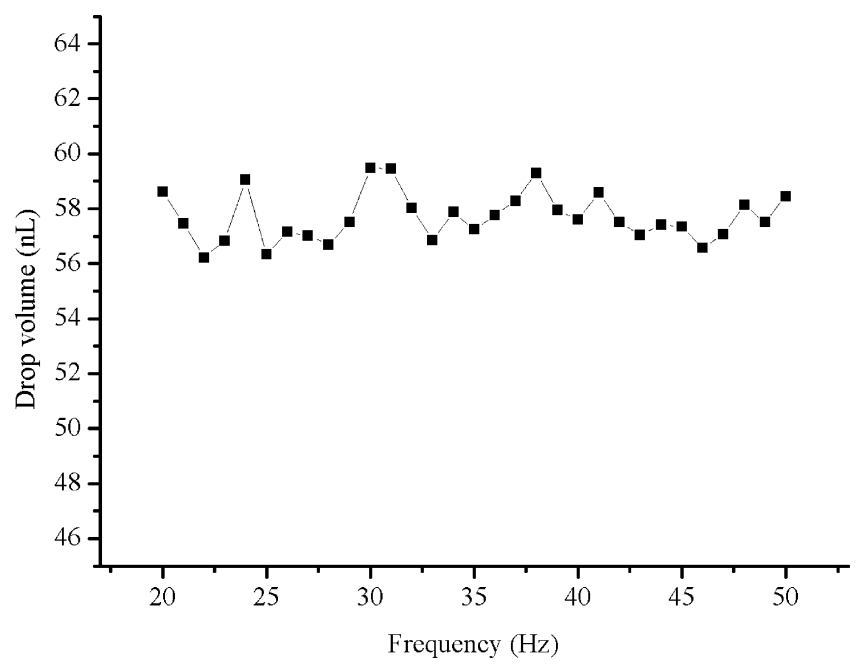

Fig. 12. The drop volume versus the applied pulse frequency.

In the ODF application, the dispensing process of a 20 -in. LCD has to be completed within $5 \mathrm{~min}$. The total amount of liquid crystal material can be estimated by the following equation:

$L=S \times d \times$ gamma

where $S$ is the display area of $\mathrm{LCD}, d$ the height of the spacer (also referred to as the cell gap) and gamma is the correction factor, which is about 1.03-1.04. The total volume is about $605 \mu \mathrm{L}$. Actuating the PZT by a frequency of $40 \mathrm{~Hz}$, when the droplet volume is $60 \mathrm{~nL}$, the total dispensing time is $252 \mathrm{~s}$, which is suitable for the ODF process.

\subsubsection{Voltage and pulse width}

The effect of the voltage on the drop volume and drop speed is shown in Fig. 13. The drop volume increases slowly when the voltage is greater than $70 \mathrm{~V}$. The effect of the pulse width on the drop volume and drop speed is shown in Fig. 14. As the pulse width increases, the drop volume increases linearly at the same driving voltage. The used nozzle size is $300 \mu \mathrm{m}$.

From experiments, two patterns of the drop formation can be classified. In the first pattern, when only one main drop forms and when it flies straight, this can be regarded as a stable ejection behavior and is named as Type 1, as shown in Fig. 15. In the second pattern, when one main drop and one satellite drop form, three types are labeled according to the flying situation of the satellite drop: (a) Type 2, if the speed of the satellite drop is greater than that of the main drop, the two drops will combine. Because the outlet velocity of the liquid is extremely high, this can help to avoid the droplet

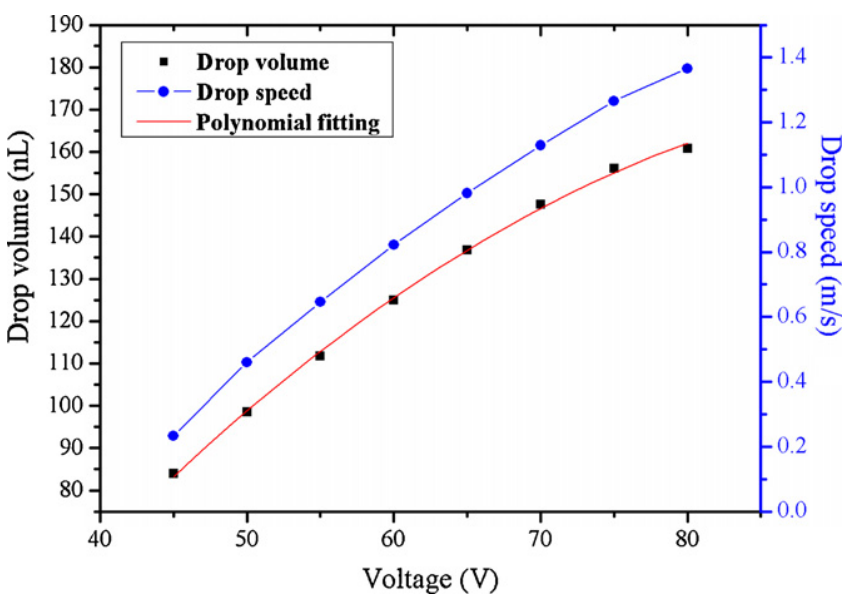

Fig. 13. Effect of the voltage on the drop volume and drop speed. Applied pulse width is $1000 \mu \mathrm{s}$.

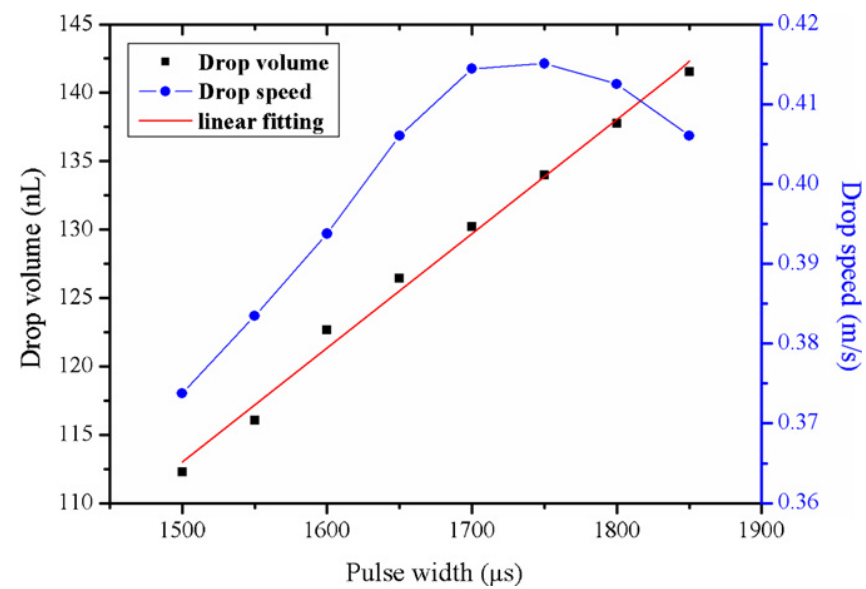

Fig. 14. Effect of the pulse width on the drop volume and drop speed. Applied voltage is $40 \mathrm{~V}$.

skew phenomenon caused by the liquid adhering on the front surface of the nozzle; (b) Type 3 , if the outlet velocity of liquid is extremely low, the satellite drop will be sucked back into the nozzle. In such a case, the route of the main drop flight is strongly affected by the environmental factors such as air fluctuations around the droplet. So, if we want to control the droplet to be deposited at the expected position of the substrate, the environmental factors must be well controlled; (c) Type 4, if the outlet velocity of liquid is medium, both drops will skew easily. This type is considered

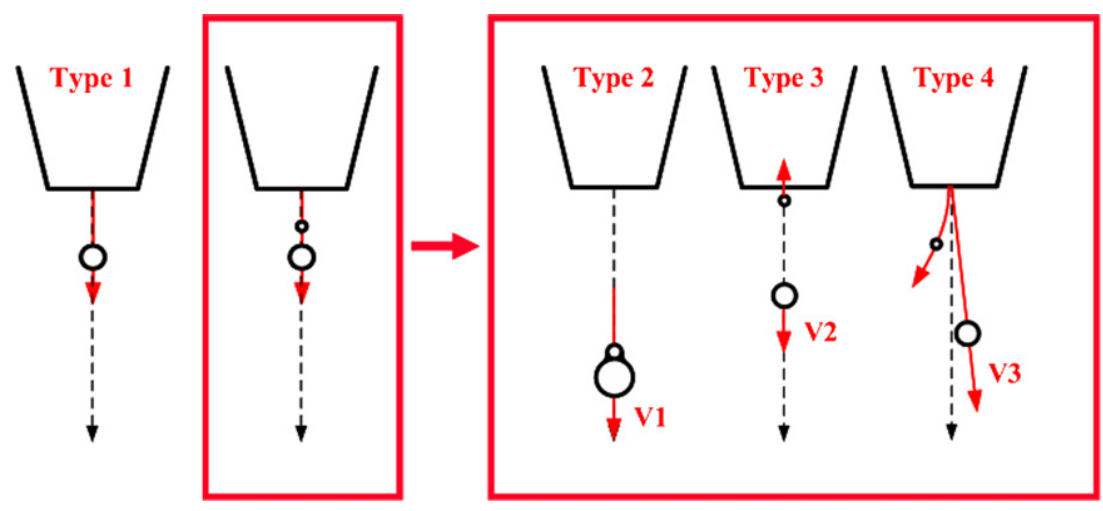

Fig. 15. Four types of ejection behavior. 


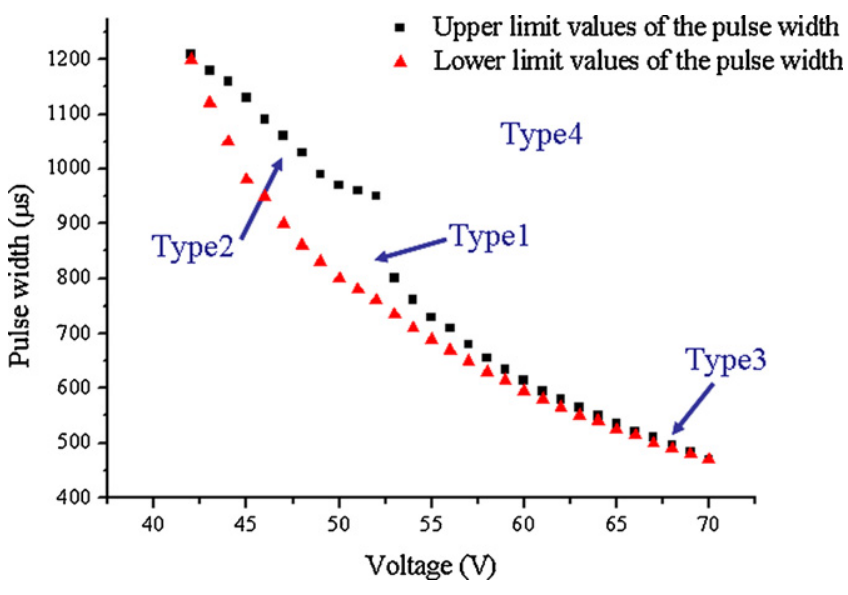

Fig. 16. The controllable range of driving parameters for a stable ejection.

the unstable ejection behavior. Types 1-3 are all regarded as stable ejection behaviors.

Under the condition of a regular voltage, we adjusted the pulse width to obtain its upper and lower limit value for a stable ejection, as shown in Fig. 16. In order to obtain the best ejection behavior, we chose the driving parameters illustrated in this figure between the lower and upper limit values of the pulse width. The optimal dwell time could be found between the lower and upper limit values of the pulse width and its corresponding voltage.

\subsubsection{The controllable drop weight}

The lower and upper limit values of the pulse width will be changed due to different kinds of liquids and nozzles. After drawing the chart, we could start to develop the calibration procedure of drop weight. First of all, we had to choose three driving parameters which were located on a line between lower and upper limit values of the pulse width, as shown in Fig. 17. Secondly, the total weights were determined after accumulating 800 drops and the data could be fitted with a quadratic function only for the voltage range of $40-48 \mathrm{~V}$, as shown in Fig. 18. Finally, the target weight of droplet could be acquired by using the corresponding driving parameters which were determined through the interpolation method by selecting the points located on the line in Fig. 17. Comparing the experimental results to the calibration data, the errors were all less than $0.12 \%$. Therefore, we could utilize this calibration procedure to

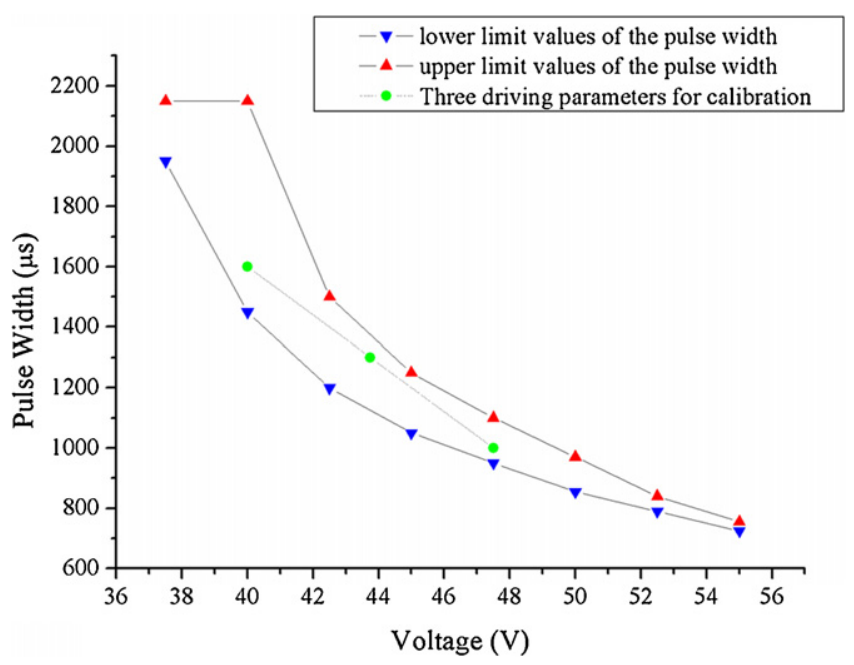

Fig. 17. Three driving parameters for weight calibration.

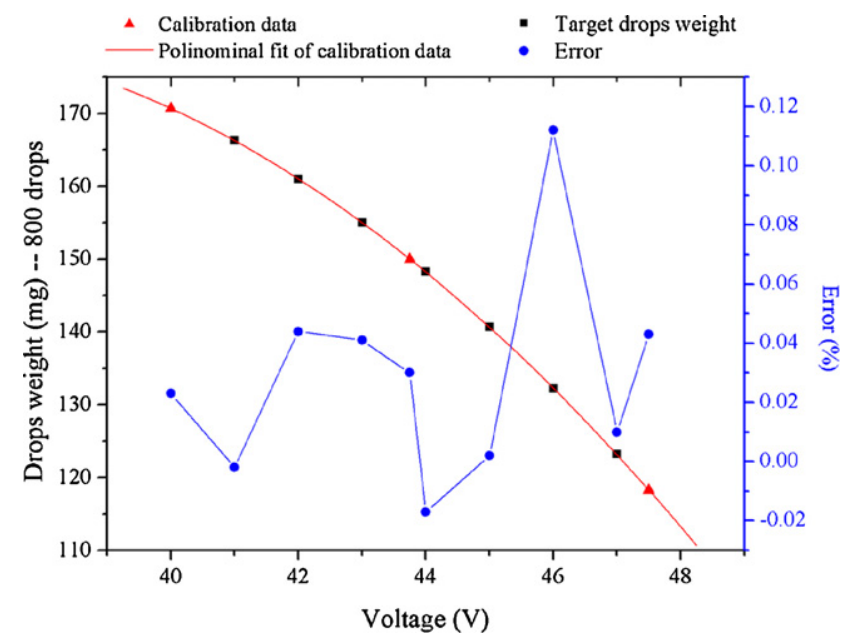

Fig. 18. Results of drop weight calibration.

achieve a goal by controlling the weight of drops. It is very useful that each time when the target weight of droplet was changed, the calibration procedure was not necessary to repeat again.

\subsection{Liquid property}

The liquid crystal material has a high viscosity and surface tension causing a difficulty in dispensing. It will easily adhere to the end face of the nozzle, and then cause the drop to skew after being ejected. During the development of the ODF, some researchers put forward particular plans to solve these problems as, e.g. using a heater to warm up the liquid directly in order to reduce its viscosity [6].

This study was focused on the drop generator, nozzle, quality of the nozzle surface and the driving conditions for a good drop performance. Because the liquid crystal has drop properties different from Newtonian fluids [20], the ejected liquid column has a longer thread, which is difficult to break up. Therefore, it can efficiently restrain the production of satellites. In other words, when the liquid crystal is ejected, the range of controllable parameters for a stable ejection is larger than that of other liquids having the same viscosity. Fig. 19 shows the drop formation sequence. The thread drains gradually into the main drop.

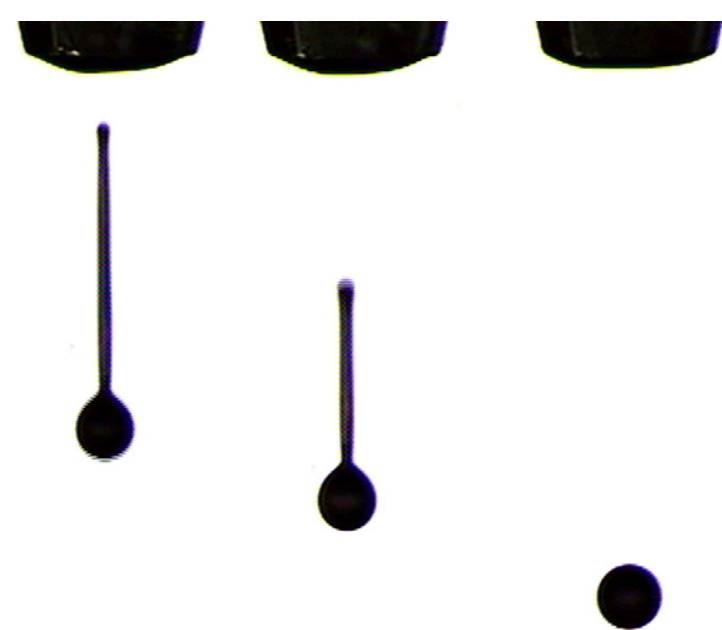

Fig. 19. The sequence of the drop formation of liquid crystals. 


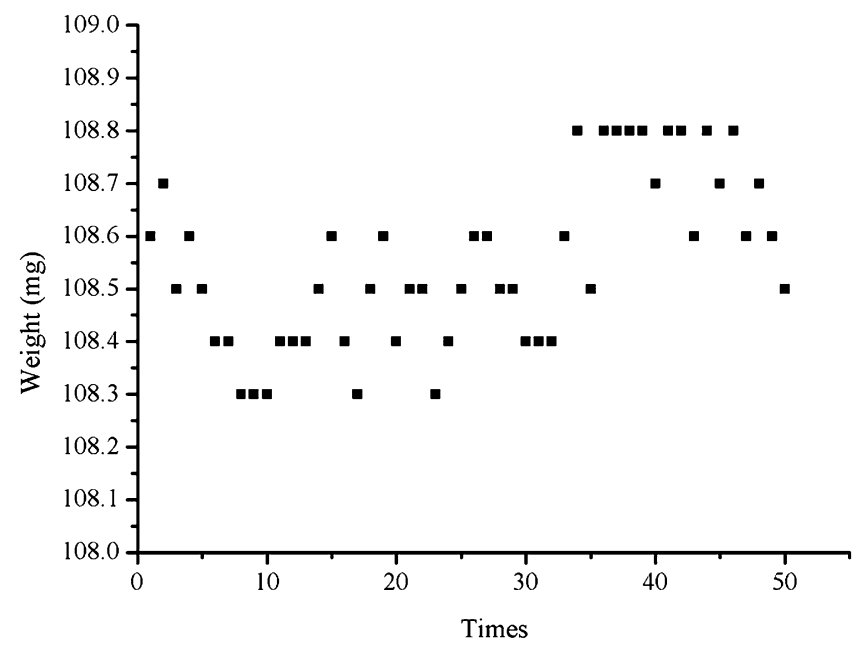

Fig. 20. Repeatability test of dispensing process.

\subsection{Repeatability}

The ODF process demands that the material of droplet generator must have a good chemical resistance and does not react with the LC material, its dispensing speed must be greater than $2 \mathrm{mg} / \mathrm{s}$, and its allowable volume variation is below $0.7 \%$. The first two requirements have been discussed in the above sections. The last goal of this work was to dispense the anticipated amount of liquid crystal. We chose the best controllable parameters and tried to keep the ambient environment steady. After accumulating 800 drops, the total weight was determined, as shown in Fig. 20. The average weight is $108.548 \mathrm{mg}$, and the variation is about $0.46 \%$. This result meets the ODF requirement.

\section{Conclusions}

Utilizing the inkjet print technology, this DOD-type droplet generator has been proved by experiments to be good in repeatability as well as precision of the drop size control. The structure of this DODtype droplet generator is simple compared to most commercial ones used in ODF processes. All the parts of this droplet generator are low cost and easy to manufacture. In addition, they can be easily assembled and dismantled and therefore cleaned handily so as to keep the liquid crystal material pure. By improving the quality of the nozzle, the slant distance of the droplet can be significantly reduced. Furthermore, by defining the four types of drop formation, we are able to find out the controllable range of driving parameters for a stable ejection between the lower and upper limit values of the pulse width. With these driving parameters, the droplet generator can be controlled easily so that the stable ejection behavior can be achieved in three types to meet the user's needs and the controllable drop weight can be realized. Under the steady-state condition of drop formation, the repeatability tests have shown highly promising results with an acceptable weight variation.

\section{Acknowledgement}

The authors gratefully acknowledge the support of the ChungShan Institute of Science and Technology for this work, through Grant No. BB96006P.

\section{References}

[1] T. Ishihara, S. Hisamitsu, H. Furukawa, Method of manufacture of liquid crystal display panel, US Patent $5,263,888$.

[2] D.H. Choo, S.U. Jung, In-line system and a method for manufacturing a liquid crystal display with special vacuum/force control, US Patent 6,657,701.
[3] H.J. Kweon, H.J. Son, Apparatus for measuring dispensing amount of liquid crystal drops and method for manufacturing liquid crystal display device using the same, US Patent 6,864,948.

[4] V. Gutfeld, R. Jacob and S.H. Lien, Method and apparatus for filling liquid crystal display (LCD) panels, US Patent 6,055,035.

[5] W.S. Kim, H.J. Kweon, H.J. Son, Liquid crystal dispensing apparatus, US Patent $6,824,023$.

[6] T. Abe, Method and dispenser for filling liquid crystal into LCD cell, US Patent $5,511,591$.

[7] Q. Liu, M. Orme, High precision solder droplet printing technology and the state-of-the-art, J. Mater. Process. Technol. 115 (2001) 271-283.

[8] M. Grove, D. Hayes, R. Cox, D. Wallace, Color flat panel manufacturing using ink jet technology, Display Works (San Jose) (1999).

[9] W. Feng, J.Y.H. Fuh, Y.S. Wong, Develpoment of a drop-on-demand micro dispensing system, Mater. Sci. Forum (Switzerland) (2006).

[10] J.C. Carter, R.M. Alvis, S.B. Brown, K.C. Langry, T.S. Wilson, M.T. McBride, M.L. Myrick, W.R. Cox, M.E. Grove, B.W. Colston, Fabricating optical fiber imaging sensors using inkjet printing technology: a pH sensor proof-of-concept, Biosens. Bioelectron. 21 (2006) 1359-1364.

[11] W.T. Pimbley, Drop formation from a liquid jet: a linear one-dimensional analysis considered as a boundary value problem, IBM J. Res. Dev. 20 (1976) 148-156.

[12] R.L. Adams, J. Roy, One dimensional numerical model of a drop-on-demand ink jet, J. Appl. Mech. 53 (1986) 193-197.

[13] S. Takahashi, H. Kitagawa, S. Tomikawa, H. Kitagawa, Y. Tomikawa, A study of liquid dispensing head using fluidic inertia, Jpn. J. Appl. Phys. 41 (2002) 3442-3445.

[14] H.C. Wu, H.J. Lin, W.S. Hwang, A numerical study of the effect of operating parameters on drop formation in a squeeze mode inkjet device, Model. Simul. Mater. Sci. Eng. 13 (2005) 17-34.

[15] J. Brunahl, A.M. Grishin, Piezoelectric shear mode drop-on-demand inkjet actuator, Sens. Actuator A: Phys. 101 (2002) 371-382.

[16] T. Laurell, L. Wallman, J. Nilsson, Design and development of a silicon microfabricated flow-through dispenser for on-line picolitre sample handling, J. Micromech. Microeng. 9 (1999) 369-376.

[17] J.D.J.S. Samuel, R. Steger, G. Birkle, R. Zengerle, P. Koltay, J. Ruhe, Modification of micronozzle surfaces using fluorinated polymeric nanofilms for enhanced dispensing of polar and nonpolar fluids, Anal. Chem. 77 (2005) 6469-6474.

[18] P.H. Chen, H.Y. Peng, H.Y. Liu, S.L. Chang, T.I. Wu, C.H. Cheng, Pressure response and droplet ejection of a piezoelectric inkjet printhead, Int. J. Mech. Sci. 41 (1999) 225-248.

[19] D.B. Bogy, F.E. Talke, Experimental and theoretical study of wave propagation phenomena in drop-on-demand inkjet devices, IBM J. Res. Dev. 28 (1976) 314-321.

[20] H.J. Shore, G.M. Harrison, The effect of added polymers on the formation of drops ejected from a nozzle, Phys. Fluids 41 (2005) 033104-1-033104-7.

\section{Biographies}

Kuang-Chao Fan was born in Taiwan, ROC, on 4 January 1950. He received the BSc degree from National Taiwan University (NTU) in 1972, MSc degree from the State University of New York at Buffalo in 1976 and PhD degree from University of Manchester Institute of Science and Technology in 1984, all in mechanical engineering. He has been a professor of mechanical engineering at National Taiwan University since August 1989. In 2003, he received the distinguished research award from National Science Council of ROC. He has promoted to the distinguished and chair professor of National Taiwan University in 2007. His research interests include manufacturing metrology, precision machining and machine tool technology. He has published around 100 journal papers and 200 conference papers. Recent topics include microcoordinate measuring machine, optical focus probe, optical switches, machine tool accuracy and tactile sensors.

Jhih-Yuan Chen received the BS degree in mechanical engineering from Chung-Yuan Christian University, Taiwan, in 2002. He received the MS degree in mechanical engineering from National Center University in 2004 and currently he is working toward the PhD degree at Nation Taiwan University. His research interests include droplet generation technology and image processing.

Ching-Hua Wang received the BS degree in mechanical engineering from National Taiwan University, Taiwan, in 1973. He received the MS degree in aerospace engineering in 1977 from Georgia Institute of Technology, Atlanta, GA. and the PhD degree in mechanical and aerospace engineering in 1983, from Northwestern University, Chicago, IL. He is currently a professor with the Department of Mechanical Engineering, National Taiwan University, where his interests are in the combustion-related researches, particularly in the liquid fuels. During the past years at Taiwan University, he successfully established a combustion laboratory, where he used the microdroplet generators studied the colliding and burning behaviors of liquid droplets. His current research topics are the energy utilization and combustion of the emulsified and the biomass and the hypergolic fuels and high velocity air-fueled impingement system (HVAF).

Wen-Chueh Pan received the PhD degree from National Taiwan Institute of Technology (NTIT) in 1983. He is currently a senior specialist of Chung-Shan Institute of Science and Technology. 